\title{
ASSOCIAÇÃO ENTRE VARIÁVEIS NUTRICIONAIS E SARCOPENIA EM LONGEVOS
}

Francine da Rocha Flores Giediel Rosa; Universidade Federal do Rio Grande do Sul; francinefflores@hotmail.com

Amanda Guterres Beuren; Universidade do Vale do Rio dos Sinos; amanda_beuren@hotmail.com

Roberta Rigo Dalla Corte; Universidade Federal do Rio Grande do Sul; robertarigod@gmail.com

Renato Gorga Bandeira de Mello, Universidade Federal do Rio Grande do Sul; renatogbmello@gmail.com

\section{RESUMO}

Introdução: No Brasil, a faixa etária com idade igual ou superior a 80 anos é a que mais cresce proporcionalmente. À medida que a população envelhece, torna-se cada vez mais evidente a necessidade do estudo dos fatores associados à sarcopenia. Objetivo: Investigar a associação entre variáveis nutricionais e sarcopenia em longevos. Metodologia: Estudo transversal, arrolou indivíduos com idade igual ou superior a 80 anos em acompanhamento ambulatorial. Para consumo alimentar, utilizou-se o recordatório alimentar de 24 horas. Estado nutricional foi categorizado conforme a Miniavaliação Nutricional. Sarcopenia foi diagnosticada através dos critérios do $E u$ ropean Working Group on Sarcopenia in Older People de 2010 e 2018. Para aferição das medidas aplicou-se o exame de Biompedância elétrica, dinamometria de preensão manual e velocidade de marcha aferida em teste de 4 metros. Resultados: A amostra foi composta por 119 longevos, população predominantemente feminina ( $\mathrm{n}=67 ; 56,3 \%)$, com média de idade de 83,4 anos $( \pm 3,0)$. As prevalências de sarcopenia variaram significativamente conforme o critério utilizado, sendo maior quando se adotou diagnóstico conforme critérios diagósticos de 2010 ou 2018 (46,7\% vs 17,6\%). Sexo feminino e baixa pontuação na Miniavaliação Nutricional aumentaram o risco para sarcopenia segundo pelo critério de 2010. Valor energético total reduzido e ingesta proteica elevada associaram-se significativamente com maior prevalência de sarcopenia e sarcopenia grave, independentemente do critério diagnóstico utilizado ou de fatores de confusão testados. Conclusão: Estado nutricional pela Miniavaliação Nutricional, consumo energético total reduzido e dieta hiperproteica, associaram-se a maior prevalência de sarcopenia.

Palavras-chave: Longevos; Sarcopenia; Miniavaliação Nutricional.

Agradecimento: O presente trabalho foi realizado com o apoio da Coordenação de Aperfeiçoamento de pessoal de Nível Superior - Brasil (CAPES). 